\title{
Interactions in low-dimensional spinor bosonic gases
}

\author{
Alexei K. Kolezhuk \\ Institute of Magnetism, National Academy of Sciences \\ and Ministry of Education of Ukraine, Kiev 03142, Ukraine \\ Institute of High Technologies, T. Shevchenko Kiev National University Kiev 01601, Ukraine \\ E-mail: kolezhuk@imag.kiev.ua \\ Received December 28, 2009
}

\begin{abstract}
Renormalization group approach for low-dimensional dilute Bose gases is generalized for the case of multicomponent bosons. As an example, systems of two- and three-component spinor bosons are considered. It is shown that interatomic interactions are strongly renormalized with a tendency to enhance the interaction symmetry.
\end{abstract}

PACS: 67.85.Fg Multicomponent condensates; spinor condensates;

67.60.Bc Boson mixtures;

37.10.Jk Atoms in optical lattices.

Keywords: ultracold atoms, spinor bosons, low dimension.

\section{Introduction}

Recently, there has been a continuing interest in multicomponent degenerate quantum gases. Multicomponent Bose condensates were initially realized in ${ }^{87} \mathrm{Rb}[1,2]$ and ${ }^{23} \mathrm{Na}$ [3] by using two different hyperfine states as different species, and later also in heteronuclear condensates of ${ }^{41} \mathrm{~K}$ and

${ }^{87} \mathrm{Rb}[4,5]$. Such systems exhibit a very rich behavior ranging from phase separation [6,7] and transitions between different magnetic states in spinor condensates [8,9] to a variety of novel transitions predicted to appear in the presence of an optical lattice [10-14]. Both in traps and optical lattices, the realization of highly anisotropic geometries («cigars» and "pancakes») is possible [15], which provides an opportunity to study the above phenomena in low-dimensional systems.

Usually, the analysis of the ground state and dynamics of multicomponent Bose systems is performed at the mean-field level, by studying coupled Gross-Pitaevskii equations [6-9]. However, it is well known that in low dimensions mean-field arguments might become inapplicable in the low density regime [16]. For instance, in a onedimensional Bose gas, with the decrease of the density, the healing length $\xi$ becomes smaller than the average interparticle distance $d$, thus invalidating the mean-field approach. In a dilute one-dimensional Bose gas, the effective interaction constant flows to strong coupling, leading to the effectively hardcore behavior of bosons $[17,18]$. In the two-dimensional Bose gas, although the ratio $\xi / d$ does not depend on the density and falls below unity only in the case of very tight two-dimensional confinement, in the dilute limit the effective coupling constant becomes strongly energy- and density-dependent $[19,20]$.
The goal of the present study is to provide a general framework for the analysis of dilute multicomponent Bose systems in the case of quasi-low-dimensional geometries. To that end, we will generalize the well-known renormalization group (RG) approach to the one-component dilute Bose gas $[17,18,21,22]$. The idea is to derive the RG equations at the critical point of the zero chemical potential (vanishing particle density), and to stop the RG flow at a certain density-dependent cutoff scale. It will be shown that interactions in a multicomponent Bose gas are in general strongly renormalized in the low density limit. For spinor bosons, the renormalization tends to enhance the interaction symmetry, effectively diminishing the spindependent part of the coupling.

\section{Renormalization group for the multicomponent Bose gas at the zero density critical point}

The starting point of our analysis will be the theory of a $N$-species bosonic field in $d$-spatial dimensions, with a general two-body (quartic) contact interaction, that effectively describes a system of atoms with typical momenta much smaller than the inverse characteristic potential range (atom size). The theory is described by the following continuum action

$$
\begin{gathered}
\mathcal{A}_{N}=\int d \tau \int d^{d} x\left\{\psi_{\alpha}^{*}\left(\partial_{\tau}-\mu_{\alpha}\right) \psi_{\alpha}+\frac{\left|\nabla \psi_{\alpha}\right|^{2}}{2 m_{\alpha}}+U\right\}, \\
U=\frac{1}{2} \sum_{\alpha \beta \alpha^{\prime} \beta^{\prime}} g_{\alpha \beta, \alpha^{\prime} \beta^{\prime}} \psi_{\alpha}^{*} \psi_{\beta}^{*} \psi_{\alpha^{\prime}} \psi_{\beta^{\prime}} .
\end{gathered}
$$

Here $\psi_{\alpha}$ are the fields describing bosonic particles with masses $m_{\alpha}, \alpha=1, \ldots N$, and for the sake of clarity we have 
set $\hbar=1$. The interaction matrix for Bose fields satisfies the obvious symmetry conditions $g_{\alpha \beta, \alpha^{\prime} \beta^{\prime}}=g_{\beta \alpha, \alpha^{\prime} \beta^{\prime}}=$ $=g_{\alpha \beta, \beta^{\prime} \alpha^{\prime}}=g_{\alpha^{\prime} \beta^{\prime}, \alpha \beta}^{*}$. The action (1) can describe a mixture in a continuum as well as in an optical lattice (in the latter case the particle density should be incommensurate with the lattice, i.e., the average occupation per lattice site should not be an integer number). The action (1) also arises in quantum magnetism problems dealing with the so-called Bose-Einstein condensation of magnons, induced by a strong external magnetic field $H$ in the vicinity of the saturation field $H_{s}$ [23]. In a frustrated magnet the magnon dispersion may have degenerate minima at inequivalent wave vectors, which gives rise to multiple «species» in an effective model [24-26], while $H_{s}-H$ plays the role of the chemical potential.

If all chemical potentials $\mu_{\alpha}$ are zero, the system is at the special critical point (the particle density vanishes). There is no self-energy correction so the full propagator just coincides with the free one $G_{\alpha \beta}(k, \omega)=$ $=\delta_{\alpha \beta} /\left(i \omega-\varepsilon_{k}^{(\alpha)}\right)$, where $\varepsilon_{k}^{(\alpha)}=k^{2} / 2 m_{\alpha}$. Interaction between the particles is, however, renormalized due to multiple scatterings. The peculiar character of the critical point leads to the fact that the contribution of any diagram containing a closed loop vanishes [27,21], so only ladder diagrams have to be included. As a result, the renormalized vertex $\Gamma$, defined at the fixed sum of external momenta $Q$, satisfies the following Bethe-Salpeter equation shown schematically in Fig. 1.

$$
\begin{gathered}
\Gamma_{\alpha \beta, \gamma \delta}(Q)=g_{\alpha \beta, \gamma \delta}-g_{\alpha \beta, \alpha^{\prime} \beta^{\prime}} f_{\alpha^{\prime} \beta^{\prime}}(Q) \Gamma_{\alpha^{\prime} \beta^{\prime}, \gamma \delta}(Q), \\
f_{\alpha \beta}(Q)=\int \frac{d^{d} k}{(2 \pi)^{d}}\left[\varepsilon_{k}^{(\alpha)}+\varepsilon_{Q-k}^{(\beta)}\right]^{-1} .
\end{gathered}
$$

Above the upper critical dimension $d=2, f_{\alpha \beta}(Q)$ converges at $Q \rightarrow 0$ and (2) simply yields the dressed (observable) interaction matrix. For $d \leq 2$ there is a singularity at $Q \rightarrow 0: f_{\alpha \beta}(Q) \propto 1 / Q$ for $d=1$ and $f_{\alpha \beta}(Q) \propto$ $\propto \ln \left(\Lambda_{0} / Q\right)$ for $d=2$, where $\Lambda_{0}$ is the ultraviolet cutoff (for a system in an optical lattice, the parameter $\Lambda_{0}$ has the physical meaning of a lattice cutoff, and in a continuum it has the sense of an inverse characteristic potential range). Thus, for $d \leq 2$ one should look at the RG flow of the running coupling matrix $\Gamma(l)=\Gamma(Q) \exp [(2-d) l]$ with the change of the scale $Q \mapsto \Lambda_{0} \exp (-l)$. Defining the matrices

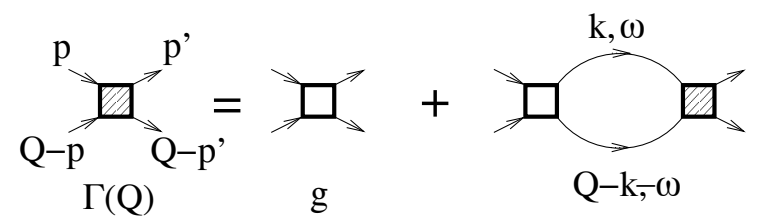

Fig. 1. The renormalized interaction vertex for the multicomponent Bose gas model (1) in the zero density limit, see Eq. (2).

$$
\begin{gathered}
\bar{\Gamma}_{\alpha \beta, \alpha^{\prime} \beta^{\prime}}=2 \Gamma_{\alpha \beta, \alpha^{\prime} \beta^{\prime}}\left(m_{\alpha \beta} m_{\alpha^{\prime} \beta^{\prime}}\right)^{1 / 2}, \\
F_{\alpha \beta, \alpha^{\prime} \beta^{\prime}}(Q)=\delta_{\alpha \beta, \alpha^{\prime} \beta^{\prime}} R_{d}\left(Q_{\alpha \beta}\right),
\end{gathered}
$$

where $m_{\alpha \beta} \equiv m_{\alpha} m_{\beta} /\left(m_{\alpha}+m_{\beta}\right)$ are the reduced masses, $Q_{\alpha \beta}=2 Q\left(m_{\alpha} m_{\beta}\right)^{1 / 2} /\left(m_{\alpha}+m_{\beta}\right)$, and

$$
R_{d}(q)=\int \frac{d^{d} k}{(2 \pi)^{d}} \frac{1}{k^{2}+q^{2}} \rightarrow\left\{\begin{array}{cc}
\frac{1}{2 q}, & d=1 \\
\frac{1}{2 \pi} \ln \frac{\Lambda_{0}}{q}, & d=2
\end{array},\right.
$$

one can write down the RG equation as

$$
\frac{d}{d l}\left\{\bar{\Gamma}(1-F \bar{\Gamma})^{-1}\right\}=0 .
$$

Further, using the identity $A X=X A=X-1$ with $X \equiv(1-A)^{-1}$ and its derivative $d X / d l=X(d A / d l) X$, those equations can be rewritten in the following form:

$$
d \bar{\Gamma} / d l+\bar{\Gamma}(d F / d l) \bar{\Gamma}=0 .
$$

For further simplification, it is convenient to redefine the interaction matrix once again. In one dimension $(d=1)$, one can introduce $\widetilde{\Gamma}=F^{1 / 2} \bar{\Gamma} F^{1 / 2}$; then, since $F$ is a diagonal matrix that for $d=1$ is proportional to $1 / Q$, one obtains $d F / d l=F$ and Eq. (5) can be rewritten as

$$
d \tilde{\Gamma} / d l=\tilde{\Gamma}-\tilde{\Gamma}^{2},
$$

which is familiar from the one-component case [28]; the only difference is that the interaction is now a matrix.

In two dimensions $(d=2)$, it is convenient to define $\tilde{\Gamma}=\bar{\Gamma} / 2 \pi$; then, using the fact that $d F / d l=1 /(2 \pi)$ for $d=2$, one can reduce (5) to

$$
d \tilde{\Gamma} / d l=-\widetilde{\Gamma}^{2} .
$$

Summarizing the above derivation, we see that the RG equations for the interaction matrix of the low-dimensional multicomponent Bose gas can be cast into the common form

$$
d \widetilde{\Gamma} / d l=(2-d) \widetilde{\Gamma}-\widetilde{\Gamma}^{2},
$$

where

$$
\begin{gathered}
\tilde{\Gamma}_{\alpha \beta, \gamma \delta}(l)=\Gamma_{\alpha \beta, \gamma \delta}(l)\left(m_{\alpha} m_{\beta} m_{\gamma} m_{\delta}\right)^{1 / 4} / \Lambda_{0}, \quad d=1, \\
\tilde{\Gamma}_{\alpha \beta, \gamma \delta}(l)=\Gamma_{\alpha \beta, \gamma \delta}(l) \sqrt{m_{\alpha \beta} m_{\gamma \delta}} / \pi, \quad d=2 .
\end{gathered}
$$

Since the $\tilde{\Gamma}$ matrices are symmetric, they can always be diagonalized by an appropriate orthogonal transformation. It is worth noting that at the zero-density critical point the Eqs (8) are exact to all orders in the interaction $\tilde{\Gamma}$, similar to the one-component case [28].

The above derivation is nothing but a direct generalization of the well-known RG approach to the one-component 
dilute Bose gas $[17,18,21,22]$. Similar to the one-component case, we will use the RG Eqs (8), derived at the critical point (zero particle density), to describe the gas of a finite but small density, and to stop the RG flow at some scale $l=l^{*}$ where the system is effectively no more dilute, i.e., where the «running» total density $\rho(l)=\rho_{\text {tot }} \mathrm{e}^{d l}$ becomes comparable with $\Lambda_{0}^{d}$. This condition determines the stopping scale $l^{*}$ as

$$
\mathrm{e}^{d l^{*}}=C_{d} \Lambda_{0}^{d} / \rho_{\text {tot }},
$$

where $C_{d=1}=2 / \pi^{2}$ and $C_{d=2}=1 / 2 \pi$ can be identified by comparing to the known results for the one-component case [22].

\section{Applications to spinor bosons}

Let us illustrate the RG approach outlined in the previous section by applying it to simple spinor bosonic gases with two and three components.

\subsection{Spin-l bosons}

Consider a gas of bosons with the hyperfine spin $F=1$. The contact interaction between two paticles depends on their total spin $S$, so there are two characteristic scattering lengths $a_{S}$ with $S=0,2$, and the interaction can be written as $[8,9]$

$$
U=\frac{1}{2}\left\{c_{0} \psi_{a}^{\dagger} \psi_{a^{\prime}}^{\dagger} \psi_{a^{\prime}} \psi_{a}+c_{2} \psi_{a}^{\dagger} \psi_{a^{\prime}}^{\dagger} F_{a b}^{\mu} F_{a^{\prime} b^{\prime}}^{\mu} \psi_{b} \psi_{b^{\prime}}-h\left(S^{z}\right)^{2}\right\}
$$

where $a, b \in\{0, \pm 1\}$ denote the three components of the bosonic field, $F^{\mu}$ are the spin-l matrices, and $S^{\mu}=$ $=\psi_{a}^{\dagger} F_{a b}^{\mu} \psi_{b}$ are the spin operators. The couplings are given by $c_{0}=\left(g_{0}+2 g_{2}\right) / 3, c_{2}=\left(g_{2}-g_{0}\right) / 3$, with $g_{S} \propto a_{S}$, and the mass $m$ is the same for all three components. The last term in (11) describes the quadratic Zeeman effect caused by an external magnetic field. The bilinear part of the quadratic Zeeman term leads to a relative shift in chemical potentials of different species, suppressing the $|0\rangle$ states for $h>0$ and $| \pm 1\rangle$ states for $h<0$, respectively. Thus, this type of interaction favors ferromagnetic spin correlations for $c_{2}<0$, and polar (nematic) ones for $c_{2}>0$ [8,9]. In addition to that, the quartic part of the Zeeman term affects interactions as well.

It is worth noting that on an optical lattice, at integer odd filling, spin- 1 bosons can be effectively described by the generalized Heisenberg model with biquadratic interactions [11,12], which also supports exotic magnetic states with nematic-type spin ordering [29,30].

The interaction (11) includes just three parameters, but the $9 \times 9$ interaction matrix $g_{a b, a^{\prime} b^{\prime}}$ contains generally five different couplings:

$$
\begin{gathered}
g_{++,++}=g_{--,--}=r, \\
2 g_{+0,+0}=2 g_{-0,-0}=f, \\
2 g_{+-,+-}=v, \\
g_{00,00}=u, \\
g_{+-, 00}=w,
\end{gathered}
$$

whose bare values are given by

$$
\begin{array}{r}
r^{(0)}=c_{0}+c_{2}-h, \quad f^{(0)}=c_{0}+c_{2}, \\
v^{(0)}=c_{0}-c_{2}+h, \quad u^{(0)}=c_{0}, \quad w^{(0)}=2 c_{2} .
\end{array}
$$

The RG equations (8) take the form

$$
\begin{gathered}
d \tilde{r} / d l=(2-d) \tilde{r}-\tilde{r}^{2}, \quad d \tilde{f} / d l=(2-d) \tilde{f}-\tilde{f}^{2}, \\
d \tilde{u} / d l=(2-d) \tilde{u}-\tilde{u}^{2}-\frac{1}{2} \tilde{w}^{2} \\
d \tilde{v} / d l=(2-d) \tilde{v}-\tilde{v}^{2}-\frac{1}{2} \tilde{w}^{2} \\
d \tilde{w} / d l=(2-d) \tilde{w}-(\tilde{u}+\tilde{v}) \tilde{w}
\end{gathered}
$$

where the «tilded» variables are defined as $\tilde{x}=x m / \Lambda_{0}$ for $d=1$ and $\tilde{x}=x m / 2 \pi$ for $d=2$.

At zero external field $(h=0)$ the $S U(2)$ symmetry dictates that the effective potential can contain only two constants $c_{0}$ and $c_{2}$, and the RG equations can be cast into the simpler form

$$
d \tilde{x} / d l=(2-d) \tilde{x}-\tilde{x}^{2},
$$

with $\tilde{x} \in\left\{\tilde{c}_{0}+\tilde{c}_{2}, \tilde{c}_{0}-2 \tilde{c}_{2}\right\}$. It is easy to see that those combinations are proportional to the scattering lengths $a_{2}$ and $a_{0}$ in the $S=2$ and $S=0$ channels, respectively. In one dimension $(d=1)$, there is a nontrivial stable fixed point $\left(\tilde{c}_{0}=1, \tilde{c}_{2}=0\right)$, which exhibits the enhanced $S U$ (3) symmetry. The other two nontrivial fixed points, $\left(\tilde{c}_{0}=2 / 3, \tilde{c}_{2}=1 / 3\right)$ and $\left(\tilde{c}_{0}=1 / 3, \tilde{c}_{2}=-1 / 3\right)$ are unstable. The corresponding RG flow for $d=1$ is shown in Fig. 2. Both for $d=1$ and $d=2$, there are two runaway flows: the one at $c_{2}>c_{0} / 2$ marks the onset of the pairing state characterized by the formation of bound singlet pairs [31-33], while the other runaway flow at $c_{2}<-c_{0}$ $\left(c_{0}>0\right)$ corresponds to the collapse instability in the ferromagnetic channel. We see that renormalization tends to enhance the interaction symmetry: the spin-dependent coupling $c_{2}$ is diminished during the RG flow.

In the more general case of a nonzero external field $h$, the RG equations can be rewritten in the same form (15) with $\tilde{x} \in\left\{\tilde{r}, \tilde{f}, \tilde{\chi}_{ \pm}\right\}$, where

$$
\chi_{ \pm}=\left(u+v \pm w \sqrt{\lambda^{2}+2}\right) / 2, \quad(u-v) / w=\lambda,
$$

and $\lambda=\left(c_{2}-h\right) / 2 c_{2}$ is a constant (an invariant of the flow) that can be controlled by the external field. The corresponding RG flow for $d=1$ is shown in Fig. 3. One can see that in the presence of external field the pairing insta- 


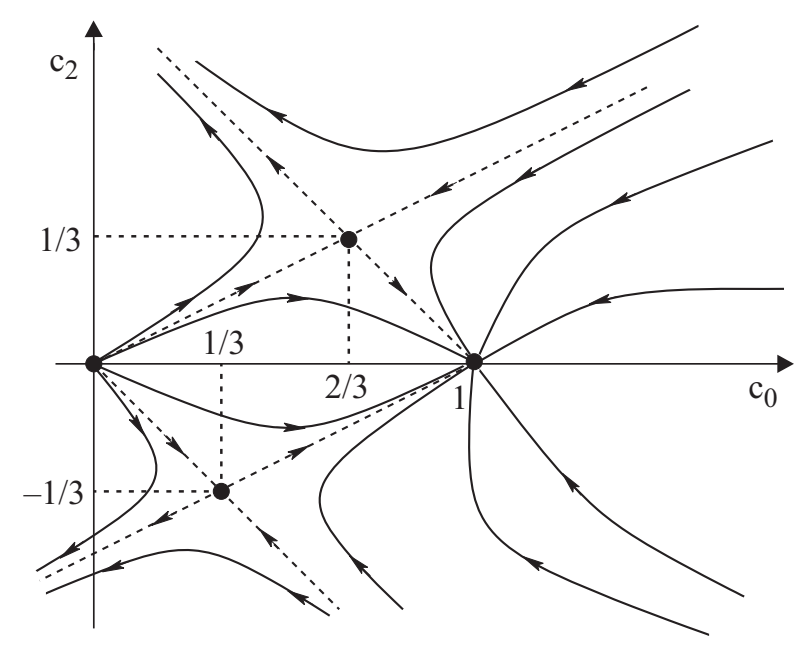

Fig. 2. The renormalization group flow diagram for the spinor boson model (11) at zero magnetic field $h=0$, for the onedimensional case.

bility point is shifted: instead of $c_{2}>c_{0} / 2$, the instability occurs at

$$
c_{2}>\left[\left(3 c_{0} / 4\right)^{2}+c_{0} h / 2\right]^{1 / 2}-c_{0} / 4 .
$$

Similarly, the ferromagnetic collapse point shifts to $c_{2}<-\left[\left(3 c_{0} / 4\right)^{2}+c_{0} h / 2\right]^{1 / 2}-c_{0} / 4$.

\subsection{Two-component gas with convertible species}

Consider now the double-species mixture with convertible species, described by the quartic interaction in (1) of the form

$$
\begin{gathered}
U\left(\psi_{1}, \psi_{2}\right)=\left(u_{11}\left|\psi_{1}\right|^{4}+u_{22}\left|\psi_{2}\right|^{4}\right) / 2+ \\
+u_{12}\left|\psi_{1}\right|^{2}\left|\psi_{2}\right|^{2}+w\left\{\left(\psi_{1}^{*}\right)^{2} \psi_{2}^{2}+\left(\psi_{2}^{*}\right)^{2} \psi_{1}^{2}\right\} / 2 .
\end{gathered}
$$

Here, only the total number of particles is conserved. Terms describing the conversion of a pair of type- 1 particles into a pair of type- 2 particles arise, for example, in models of bosons in optical lattices with considerably populated higher

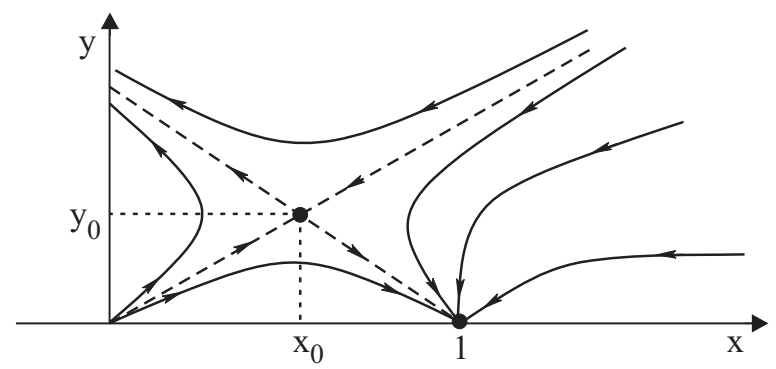

Fig. 3. The RG flow diagram for the spinor boson model (11) in one dimension, for the general case of nonzero external field $h$. Here $x=(\tilde{u}+\tilde{v}) / 2, y=\tilde{w}$, and $\left(x_{0}, y_{0}\right)=\left(1 / 2,1 / \sqrt{2+\lambda^{2}}\right)$. The flow is symmetric against the $x$-axis. bands [34]. In that case, the effective masses of the two species, as well as the intraspecies interactions, might differ. In frustrated magnets pair conversion terms may arise if the magnon dispersion has two degenerate minima at such incommensurate wave vectors $\mathbf{q}_{1}, \mathbf{q}_{2}$ that $2\left(\mathbf{q}_{1}-\mathbf{q}_{2}\right)=\mathbf{g}$, where $\mathbf{g}$ is the reciprocal lattice vector.

In the notation of (1), the bare couplings are $g_{11,11}=u_{11}$, $g_{22,22}=u_{22}, g_{12,12}=u_{12} / 2$, and $g_{11,22}=w$. Denoting the entries of the $\widetilde{\Gamma}$ matrix by $\tilde{u}_{11}$, etc., we can write the RG Eqs (8) in the form (15) with $\tilde{x} \in\left\{\tilde{u}_{12}, \tilde{u}_{+}, \tilde{u}_{-}\right\}$, where

$$
\begin{gathered}
\tilde{u}_{ \pm}=\tilde{u} \pm \widetilde{W}, \quad \widetilde{W}^{2}=\tilde{w}^{2}+\tilde{s}^{2}, \\
\tilde{u}=\left(\tilde{u}_{11}+\tilde{u}_{22}\right) / 2, \quad \tilde{s}=\left(\tilde{u}_{11}-\tilde{u}_{22}\right) / 2 .
\end{gathered}
$$

The tilded variables for $d=1$ are defined as $\widetilde{w}=w \sqrt{m_{1} m_{2}} / \Lambda_{0}$ and $\tilde{u}_{\alpha \beta}=u_{\alpha \beta} \sqrt{m_{\alpha} m_{\beta}} / \Lambda_{0}$; for $d=2$ one has $\tilde{w}=w \sqrt{m_{1} m_{2}} / 2 \pi$ and $\tilde{u}_{\alpha \beta}=u_{\alpha \beta} m_{\alpha \beta} / \pi$. The ratio $\tilde{s} / \tilde{w}$ remains constant during the RG flow. For $d=1$, the flow diagram has the same form as shown in Fig. 3; in this case $x=\tilde{u}, y=\widetilde{W}$, and the unstable fixed point is at $\left(x_{0}, y_{0}\right)=(1 / 2,1 / 2)$. Again, the RG flow tends to enhance the symmetry: it is easy to see that at the stable fixed point the potential (18) becomes $S U(2)$-symmetric,

$$
U\left(\psi_{1}, \psi_{2}\right) \rightarrow \frac{\Lambda_{0}}{2}\left(\frac{\left|\psi_{1}\right|^{2}}{\sqrt{m_{1}}}+\frac{\left|\psi_{2}\right|^{2}}{\sqrt{m_{2}}}\right)^{2} .
$$

Both for $d=1$ and $d=2$, there are «runaway» flows at $|\widetilde{W}|>\tilde{u}$, which translates into the condition

$$
w^{2}>u_{11} u_{22}
$$

By the analogy with spin-1 bosons, one may assume that those runaways correspond to the pairing instability.

\section{Summary}

Renormalization group approach for low-dimensional dilute Bose gases is generalized for the case of multicomponent bosons. The approach is applied to systems of twoand three-component spinor bosons. It is shown that interatomic interactions are strongly renormalized with a tendency to enhance the interaction symmetry.

Support by Deutsche Forschungsgemeinschaft (the Heisenberg Program, KO 2335/1-2) is gratefully acknowledged.

1. C.J. Myatt, E.A. Burt, R.W. Ghrist, E.A. Cornell, and C.E. Wieman, Phys. Rev. Lett. 78, 586 (1997).

2. D.S. Hall, M.R. Matthews, J.R. Ensher, C.E. Wieman, and E.A. Cornell, Phys. Rev. Lett. 81, 1539 (1998).

3. D.M. Stamper-Kurn, M.R. Andrews, A.P. Chikkatur, S. Inouye, H.-J. Miesner, J. Stenger, and W. Ketterle, Phys. Rev. Lett. 80, 2027 (1998); J. Stenger, S. Inouye, D.M. 
Stamper-Kurn, H.J. Miesner, A.P. Chikkatur, and W. Ketterle, Nature 396, 345 (1998).

4. G. Modugno, M. Modugno, F. Riboli, G. Roati, and M. Inguscio, Phys. Rev. Lett. 89, 190404 (2002).

5. J. Catani, L. De Sarlo, G. Barontini, F. Minardi, and M. Inguscio, Phys. Rev. A77, 011603(R) (2008).

6. T.-L. Ho and V.B. Shenoy, Phys. Rev. Lett. 77, 3276 (1996).

7. E. Timmermans, Phys. Rev. Lett. 81, 5718 (1998).

8. T.-L. Ho, Phys. Rev. Lett. 81, 742 (1998).

9. T. Ohmi and K. Machida, J. Phys. Soc. Jpn. 67, 1822 (1998).

10. A. Kuklov, N. Prokof'ev, and B. Svistunov, Phys. Rev. Lett. 92, 050402 (2004).

11. F. Zhou and M. Snoek, Ann. Phys. 308, 692 (2003).

12. A. Imambekov, M. Lukin, and E. Demler, Phys. Rev. A68, 063602 (2003).

13. E. Altman, W. Hofstetter, E. Demler, and M.D. Lukin, New J. Phys. 5, 113 (2003).

14. A. Isacsson, Min-Chul Cha, K. Sengupta, and S.M. Girvin, Phys. Rev. B72, 184507 (2005).

15. For a review see, e.g., I. Bloch, J. Dalibard, and W. Zwerger, Rev. Mod. Phys. 80, 885 (2008).

16. See, e.g., Chapter 17 of L. Pitaevskii and S. Stringari, BoseEinstein Condensation, Oxford University Press, (2003).

17. D.S. Fisher and P.C. Hohenberg, Phys. Rev. B37, 4936 (1988).

18. D.R. Nelson and H.S. Seung, Phys. Rev. B39, 9153 (1989).
19. D.S. Petrov, M. Holzmann, and G.V. Shlyapnikov, Phys. Rev. Lett. 84, 2551 (2000).

20. M.D. Lee, S.A. Morgan, M.J. Davis, and K. Burnett, Phys. Rev. A65, 043617 (2002).

21. M.P.A. Fisher, P.B. Weichman, G. Grinstein, and D.S. Fisher, Phys. Rev. B40, 546 (1989).

22. E.B. Kolomeisky and J.P. Straley, Phys. Rev. B46, 11749 (1992).

23. E.G. Batyev and L.S. Braginskii, Zh. Eksp. Teor. Fiz. 87, 1361 (1984) [Sov. Phys. JETP 60, 781 (1984)].

24. T. Nikuni and H. Shiba, J. Phys. Soc. Jpn. 64, 3471 (1995).

25. G. Jackeli and M.E. Zhitomirsky, Phys. Rev. Lett. 93, 017201 (2004).

26. A. Kolezhuk and T. Vekua, Phys. Rev. B72, 094424 (2005).

27. D. Uzunov, Phys. Lett. A87, 11 (1981).

28. S. Sachdev, Quantum Phase Transitions, Cambridge University Press (1999).

29. V.G. Baryakhtar, V.V. Gann, and D.A. Yablonsky, Fiz. Tverd. Tela 17, 1744 (1975).

30. N. Papanicolaou, Phys. Lett. A116, 89 (1986).

31. J. Cao, Y. Jiang, and Y. Wang, EPL 79, 30005 (2007).

32. F.H.L. Essler, G.V. Shlyapnikov, and A.M. Tsvelik, J. Stat. Mech. Theor. Exp., P02027 (2009).

33. K. Yang, preprint arXiv:0907.4739.

34. J. Larson, A. Collin, and J.-P. Martikainen, Phys. Rev. A79, 033603 (2009). 